\title{
A FEASIBILITY STUDY ON THE MEASUREMENT OF TREE TRUNKS IN FORESTS USING MULTI-SCALE VERTICAL IMAGES
}

\author{
A. Berveglieri ${ }^{\text {a }}$, R. A. Oliveira ${ }^{\text {a }}$, A. M. G. Tommaselli ${ }^{\mathrm{b}}$ \\ ${ }^{\mathrm{a}, \mathrm{b}}$ Univ Estadual Paulista - UNESP, Faculty of Sciences and Technology, Presidente Prudente, Brazil, \\ ${ }^{\mathrm{a}}$ Graduate Program in Cartographic Sciences, ${ }^{\mathrm{b}}$ Department of Cartography \\ a adilsonberveg@gmail.com, ${ }^{\mathrm{a}}$ raquel88@gmail.com, ${ }^{\mathrm{b}}$ tomaseli@ fct.unesp.br
}

KEY WORDS: Photogrammetry, Orientation, Matching, Circle Fitting, Fisheye, Bundle Adjustment

\begin{abstract}
:
The determination of the Diameter at Breast Height (DBH) is an important variable that contributes to several studies on forest, e.g., environmental monitoring, tree growth, volume of wood, and biomass estimation. This paper presents a preliminary technique for the measurement of tree trunks using terrestrial images collected with a panoramic camera in nadir view. A multi-scale model is generated with these images. Homologue points on the trunk surface are measured over the images and their ground coordinates are determined by intersection of rays. The resulting XY coordinates of each trunk, defining an arc shape, can be used as observations in a circle fitting by least squares. Then, the DBH of each trunk is calculated using an estimated radius. Experiments were performed in two urban forest areas to assess the approach. In comparison with direct measurements on the trunks taken with a measuring tape, the discrepancies presented a Root Mean Square Error (RMSE) of $1.8 \mathrm{~cm}$ with a standard deviation of $0.7 \mathrm{~cm}$. These results demonstrate compatibility with manual measurements and confirm the feasibility of the proposed technique.
\end{abstract}

\section{INTRODUCTION}

Estimating the volume of wood in forest areas or reforestation is an important task for fulfilling several purposes, e.g., monitoring of tree growth, forest inventory, and biomass estimation. For this task, samples in forest areas are systematically collected to be used as a control.

Nowadays, on-site ground survey is required with manual measurements at $1.30 \mathrm{~m}$ height, also known as Diameter at Breast Height $(\mathrm{DBH})$, which is a time-consuming task and which produces only partial knowledge of the forest structure. This parameter is typically used together with other variables to obtain a biomass estimate in forests. Other techniques are also employed to collect forest data, such as laser scanning, RADAR, or fisheye stereo models, but these have disadvantages due to their cost or limitations in the field of view.

This paper presents a feasibility study for the automation of a data acquisition process using panoramic optical images. A technique based on fisheye lens is used to collect multi-scale images in forest areas. Significant variables can be obtained from the scenes, which include several samples of diameters along the trunk, ground texture, shadows as well as the position and spatial distribution of the trees. Furthermore, the acquired images are a permanent record of the sampled area, enabling error assessment and, later, data analysis.

Some studies on the use of fisheye cameras in forest survey will be presented in the following sections, as well as the proposed technique, and the results of the preliminary trials.

\section{BACKGROUND}

Reliable measurements of the volume of wood in forest environments can provide essential information about forests. Commonly, direct and indirect methods are the two main categories of procedure to estimate forest parameters (Jonckheere et al., 2004). Although less accurate, indirect methods are less labor intensive and less expensive than direct methods. Indirect methods based on image measurements have been developed to measure tree attributes, such as height, diameter and volume (Hapca et al., 2007; Forsman et al., 2012) and to estimate the transmission of solar radiation through the forest canopy (Jonckheere et al., 2004). These parameters are typically used to understand how forests grow and develop.

The work by Hapca et al. (2007) used two digital photographs taken from two stations with convergence defining an intersection angle of $90^{\circ}$ to reconstruct the $3 \mathrm{D}$ shape of standing trees. Forsman et al. (2012) proposed a methodology to estimate DBH with a system composed of five Canon digital cameras attached to a calibrated rig. The developed method generated point clouds using Scale-Invariant Feature Transform (SIFT) technique in multiple images in combination with epipolar line geometry. Then, projection of the point cloud to a simulated ground plane was followed by circle-fitting to the remaining points. Forsman et al. (2012) obtained Root Mean Square Error (RMSE) of $2.88 \mathrm{~cm}$ for trees located up to a distance of $7 \mathrm{~m}$.

Fisheye systems increase the possibility of obtaining images in places of difficult access and allow a large ground coverage area. This sensor is more compact and simpler than a system with multiple cameras, reducing the time demanded in equipment calibration and possible errors propagated in this process. Hemispherical images of forest canopy have been widely used since Evans and Coombe (1959) showed that images acquired using cameras with fisheye lens could be used to study sunlight penetration through forest environments by superimposing the track of the sun on the images. Fisheye optics systems provide hemispherical images with a large field of view around the camera using only one image, but the images have large scale variations caused by the imaging geometry and, 
consequently, loss of resolution. The camera must be accurately calibrated using a specific model for the fisheye lens (Schneider et al., 2009; Tommaselli et al., 2014).

In order to obtain 3D information from multiple fisheye images, correspondence techniques commonly used in perspective cameras need to be adapted to consider their non-collinear geometry (Herrera et al., 2011; Tommaselli and Berveglieri, 2014). Herrera et al. (2011) proposed a fisheye stereovision method for forest environments combining a step for segmentation of images (to separate textures of interest) with the matching process. The final decision about the correct match is made based on a weighted Fuzzy similarity approach and the objective of the methodology is to compute disparity maps of the tree trunks.

In the following sections, a technique also based on fisheye lens will be presented to measure the DBH of tree trunks indirectly. The method uses multi-scale models and circle fitting by least-squares algorithm. Experiments with seven tree trunks were performed to assess the approach, and the first results demonstrated the feasibility of the proposed technique.

\section{METHODOLOGY}

In general, many trees have trunks with cylindrical shape, which are used as a reference for several studies on forests. From this observation, and since the trees typically present vertical growth, the primary idea is to locate distinguishable points on the trunk surface to determine their ground coordinates around a height of $1.3 \mathrm{~m}$. As depicted in Figure 1, planimetric coordinates referring to the trunk surface can be used to adjust a circumference or circle and to estimate its diameter or radius.

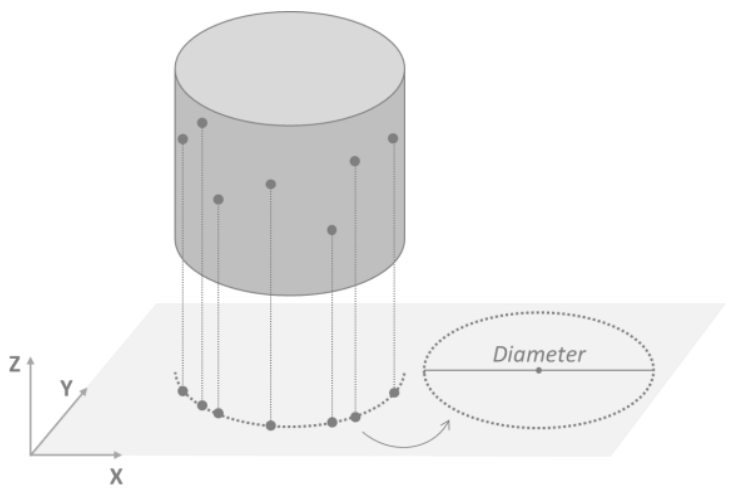

Figure 1. Arc described by the planimetric coordinates of the points on the trunk. A circle can be fitted to determine its diameter.

The entire process consists of the following steps: camera calibration, image acquisition, feature extraction, image matching, bundle adjustment, determination of coordinates in the object space, and circle adjustment.

\subsection{Camera calibration}

The first step is the camera calibration to determine the Interior Orientation Parameters (IOPs). Usually the mathematical model is based on the collinearity equations and includes the lens distortion parameters by the Conrady-Brown model (Brown, 1971). However, the image acquisition with fisheye lens does not follow the collinearity conditions due to its internal geometry and thus, a suitable model should be used, as the equidistant, equisolid-angle or orthographic model (Schneider et al., 2009). The linearized equation system can be solved using least-squares method and considering certain constraints imposed on the ground coordinates, object distances, or EOP observations.

\subsection{Image acquisition}

The image acquisition technique, designed by Tommaselli and Berveglieri (2014), uses a digital camera with wide-angle or fisheye lens attached to a telescopic pole, which is positioned among the trees to acquire vertical images from the sampling sites. Images with nadir perspective are acquired in set positions $(\mathrm{X}, \mathrm{Y})$ at different heights (ranging between 3.5 and $5.0 \mathrm{~m}$ above the ground), as shown in Figure 2.

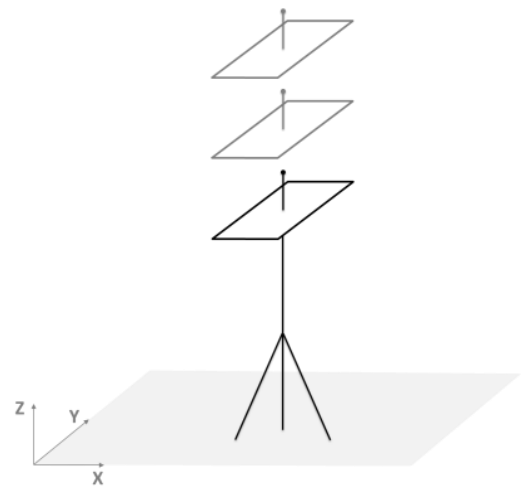

Figure 2. Acquisition of vertical images at different heights.

Using the strategy of vertical camera displacement ensures that the same trees are visible in all images without significant changes in trunk aspect, which is not guaranteed if a horizontal displacement is carried out, when many occlusions are likely to occur.

\subsection{Feature extraction and image matching}

A feature-based matching algorithm using SIFT is applied to find homologue points, which are used as tie points to ensure geometric robustness. Distinguishable points on the ground and trunks are extracted and used to estimate the Exterior Orientation Parameters (EOPs) of each camera station.

SIFT is an image processing technique based on local gradients. The extracted features are partially invariant to changes in scale, rotation, illumination, and camera viewpoint. SIFT descriptors are computationally generated to describe images. Full details on SIFT can be found in Lowe (2004).

The presence of shadows is a characteristic of the interior of forests. It can happen that homologue points are obtained near a shadow border which is moving over time and this can lead to false matches in successive images. To avoid this type of point, an algorithm was used to label shadow areas and check each tie point concerning its neighbourhood. Tie points within shadow border are discarded.

\subsection{Bundle adjustment of the multi-scale model}

The set of images forms a multi-scale model that is used to determine the $3 \mathrm{D}$ coordinates of the tie points in the object space, without control points. To achieve this, the EOPs must be estimated with accurate values, and the image matching must be established with reliable matches. The heights of the camera 
stations are accurately measured during image acquisition to be used as constraints in the image orientation process. The attitude parameters are considered as unknowns.

The images have approximately the same planimetric position and attitude, but different heights, which causes scale changes in the images. The EOPs of the lowest image are considered fixed (six absolute constraints). For the EOPs of the second and third images, constraints are also imposed with respect to height and small variations due to vertical displacement of the pole (1 $\mathrm{mm}$ in height and $5 \mathrm{~cm}$ in planimetry). Then, the multi-scale model can be oriented with bundle adjustment using an arbitrary local reference system.

Bundle adjustment uses the least-squares method to solve a system of non-linear equations in which the image coordinates are the observations and the EOPs and ground coordinates of the tie points are the unknowns. In the case of this technique, the combined adjustment method for bundle adjustment was used with the equidistant model for fisheye lenses (Tommaselli and Berveglieri, 2014).

\subsection{Circle fitting}

Points on the trunk surface should be measured and matched in the images. Inserting them as tie points in the bundle adjustment yields a set of coordinates in the object space. The planimetric coordinates obtained from the trunk describe a curve that can be fitted with a circumference or circle by least-squares adjustment.

A circle can be specified by its centre $\left(\mathrm{X}_{0}, \mathrm{Y}_{0}\right)$ and its radius $r$. However, the mathematical model is a non-linear equation that must be linearized, and the least-squares approach can be used to estimate the parameters from redundant observations. The $\mathrm{XY}$ ground coordinates of points (around $\mathrm{DBH} \pm 0.3 \mathrm{~m}$ ) are used as observations whereas $\mathrm{X}_{0}, \mathrm{Y}_{0}$ and $r$ are the parameters to be estimated. A minimum of three points (or observations) nonvertically aligned is required, as well as approximate initial values for the parameters. The solution is achieved using leastsquares adjustment computed by the parametric or combined methods (Vaníček and Wells, 1972). After iterative algorithm convergence, the best circle is fitted to determine its parameters. Then, the trunk diameter can be calculated.

\section{EXPERIMENTS AND RESULTS}

Preliminary experiments were performed in urban forest areas to check the feasibility of the proposed technique. The following subsections will present the entire development of the steps for DBH measurement of the considered trunks.

\subsection{Preparation of data}

A Fuji S3Pro digital camera with Bower fisheye lens was used to acquire the images. First, calibration was performed in a $3 \mathrm{D}$ calibration field composed of coded targets of ARUCO style (Garrido-Jurado et al., 2014), regularly arranged on the floor and walls.

Figure 3 shows one of the 11 images used in the calibration process. The target corners were automatically located resulting in 680 observations generated from 340 ground points. The Calibration Multi-Camera (CMC) software, developed in-house by Ruy et al. (2009) with adaptation for the equidistant model by Tommaselli et al. (2014), was used to calibrate the fisheye lens. The results of camera calibration are summarized in Table 1.

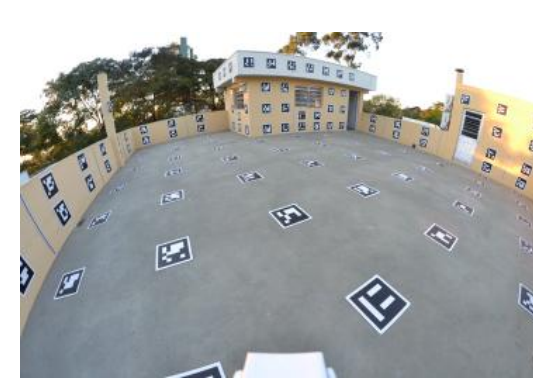

(a)

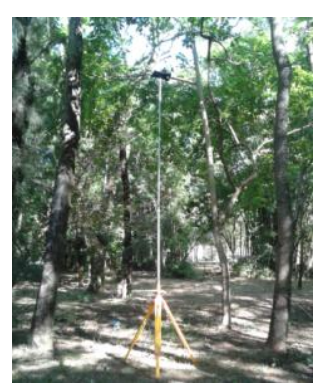

(b)
Figure 3. (a) Example of a fisheye image used in the 3D calibration field; (b) Camera positioned among trees to collect vertical images.

\begin{tabular}{c|c|c}
\hline Parameter & Estimated value & Estimated standard deviation \\
\hline $\mathrm{f}(\mathrm{mm})$ & 8.3846 & $0.0064( \pm 1.18$ pixels $)$ \\
\hline $\mathrm{x}_{0}(\mathrm{~mm})$ & 0.1014 & $0.0059( \pm 1.09$ pixels $)$ \\
\hline $\mathrm{y}_{0}(\mathrm{~mm})$ & -0.3027 & $0.0043( \pm 0.79$ pixels $)$ \\
\hline $\mathrm{K}_{1}\left(\mathrm{~mm}^{-2}\right)$ & $4.27 \times 10^{-4}$ & $1.36 \times 10^{-5}$ \\
\hline $\mathrm{K}_{2}\left(\mathrm{~mm}^{-4}\right)$ & $7.99 \times 10^{-7}$ & $1.57 \times 10^{-7}$ \\
\hline $\mathrm{K}_{3}\left(\mathrm{~mm}^{-6}\right)$ & $-2.08 \times 10^{-9}$ & $6.07 \times 10^{-10}$ \\
\hline $\mathrm{P}_{1}\left(\mathrm{~mm}^{-1}\right)$ & $1.63 \times 10^{-5}$ & $4.04 \times 10^{-6}$ \\
\hline $\mathrm{P}_{2}\left(\mathrm{~mm}^{-1}\right)$ & $-5.47 \times 10^{-5}$ & $9.78 \times 10^{-6}$ \\
\hline$\sigma$ naught & 0.92 & (considering $\sigma$ a-priori $=1)$ \\
\hline
\end{tabular}

Table 1. IOPs estimated by the camera calibration using bundle adjustment with the equidistant model.

Vertical images were collected at two sites, as presented in Figure 4, in which the trunks used were numbered for identification purposes.
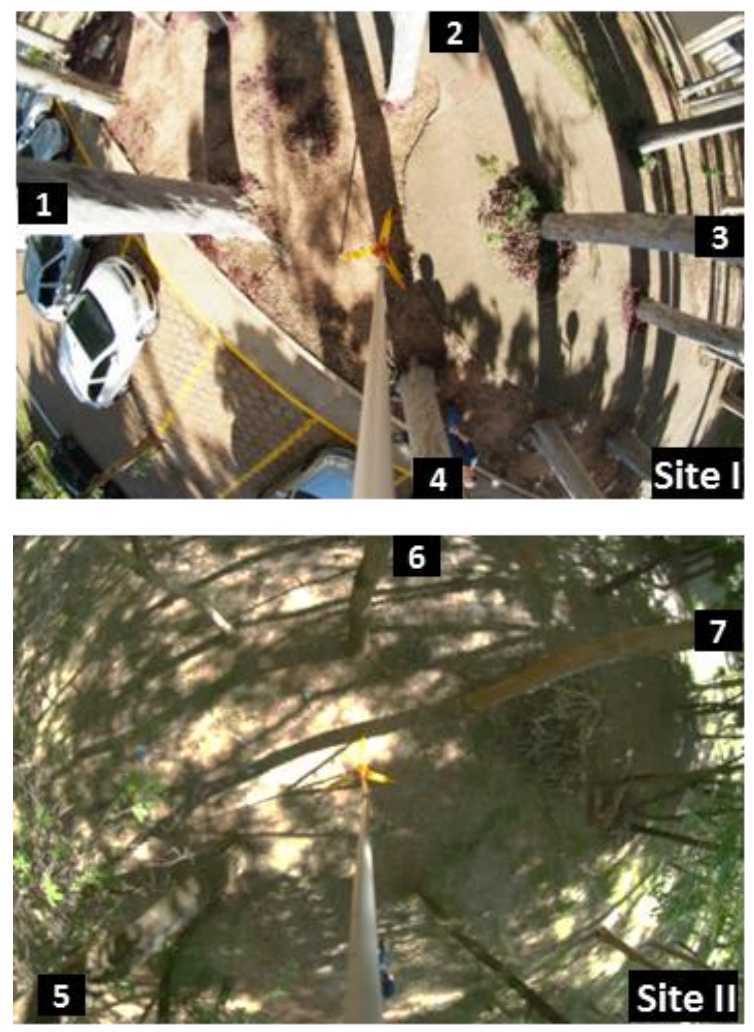

Figure 4. Fisheye vertical images collected from two sites with identification of the trunks used in the experiments. 
Tie points were automatically extracted and matched based on SIFT descriptors, as shown in Figure 5. Several additional points on trunk profiles were manually measured across the images to have their coordinates determined in the object space.

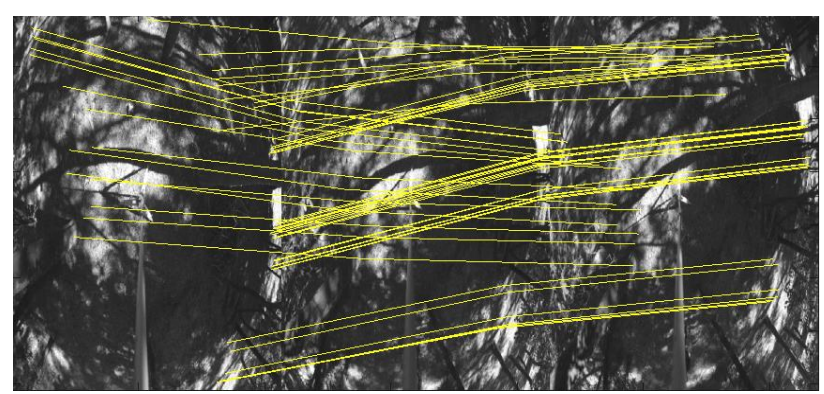

Figure 5. Matches using SIFT descriptors extracted among three multi-scale images.

A Matlab script, developed by Lowe (2005), was used to compute SIFT descriptors. Further filtering techniques were also implemented to select and match the descriptors under verification of shadow borders.

\subsection{Bundle adjustment}

Bundle adjustment calculations were performed with CMC software, in which the equidistant model was implemented. The IOPs were previously estimated in the calibration step, and initial EOPs, image coordinates of tie points, were measured. An arbitrary reference system was defined considering the planimetric position of the telescopic pole as the origin. The ground coordinates of the tie points will be determined in this local reference system. The image coordinates of the tie points, extracted with SIFT technique, were inserted in the photogrammetric project as observations with a standard deviation of $\sigma=0.5$ pixels.

The initial values of the EOPs, inserted as weighted constraints, were based on direct measurements during image acquisition. The constraint weights were based on assumptions of the variations of the camera station and relative accuracy of the direct measurements:

- Six absolute constraints were imposed on the EOPs of the first image (the lowest defining the origin and orientation of a local Cartesian reference system);

- The height of the second image was constrained with a standard deviation of $\sigma=1 \mathrm{~mm}$, and the XY coordinates of the perspective centre were constrained with a standard deviation of $\sigma=5 \mathrm{~cm}$, because small variations are expected due to the vertical displacement of the pole. The attitude parameters were configured with a standard deviation of $\sigma=30^{\circ}$, which does not affect the results. The lever arm was previously determined in the calibration step;

- The EOPs of the third image were similarly constrained. Initial values for $\mathrm{X}_{0}, \mathrm{Y}_{0}$ and $r$ were automatically estimated based on centroids of the point coordinates over each trunk.

In the bundle adjustment, the EOPs of the three images and the $3 \mathrm{D}$ coordinates of the tie points were simultaneously estimated. After this orientation step, horizontal profiles of the trunks at several different heights can be extracted provided that enough samples (tie points) are available in the suitable area. In some cases, the SIFT technique was not able to find enough points in the target area and manual measurements were performed to define additional tie points. In a future work this search for dense point cloud will be carried out by a further matching technique.

\subsection{Results with the circle fitting}

Figure 6 shows the four circles fitted over the tie points representing some trunks of Site I used in the experiments.

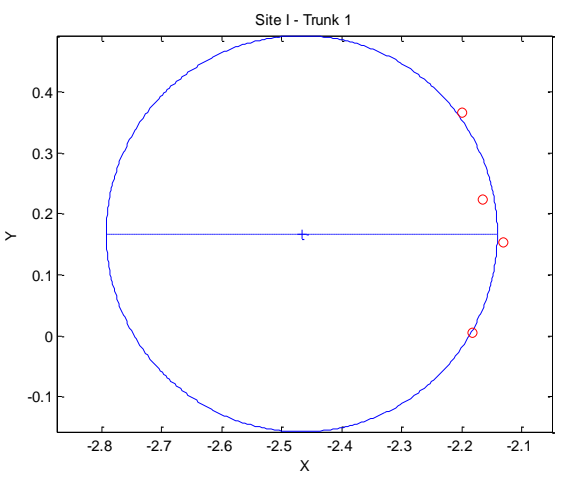

(a)

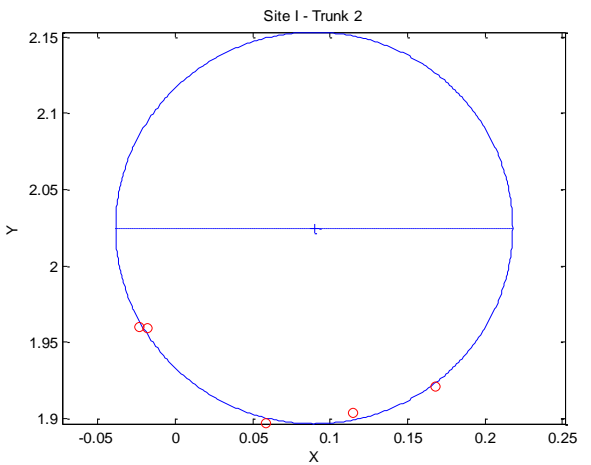

(b)

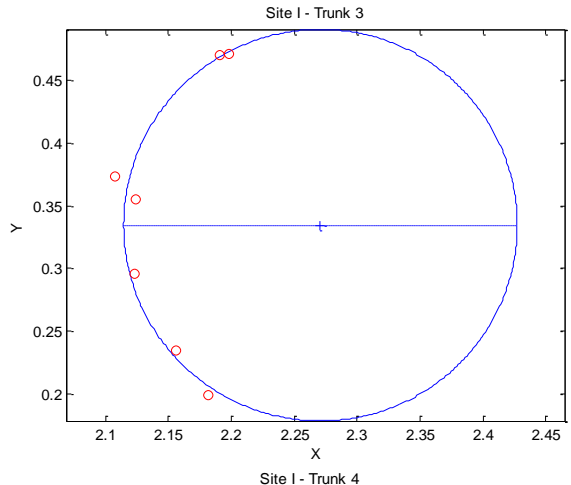

(c)

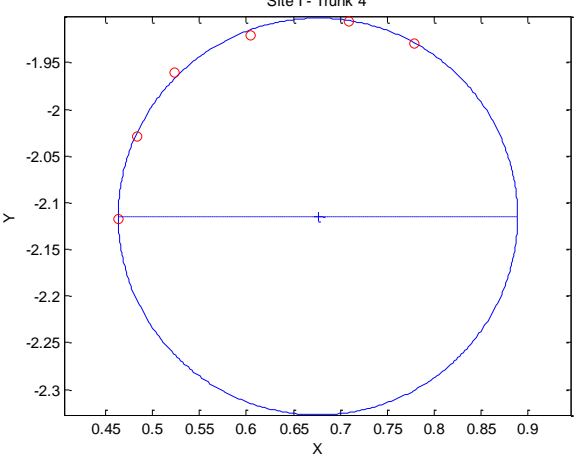

Figure 6. Circle fitted to the XY coordinates of points on the trunk surface (small red circles). 
The set of suitable object points (tie points in a neighbourhood of DBH, $1.3 \mathrm{~m} \pm 0.3 \mathrm{~m}$ in height) on each trunk was used to adjust a circle by least squares, from which each radius was determined.

A major problem with all indirect techniques is that only part of each trunk is visible from the camera viewpoint. However, the acquisition of points covering as much as possible of the width of the trunk makes it possible to obtain its curvature to fit a circle.

In Figure 6, the small red circles represent the planimetric coordinates of tie points around the DBH estimated in the bundle adjustment. In Figures 6 and 7, both the estimated point coordinates and the circles fitted to these points can be observed. The same procedure was performed for Site II considering three trunks, and the results are shown in Figure 7.
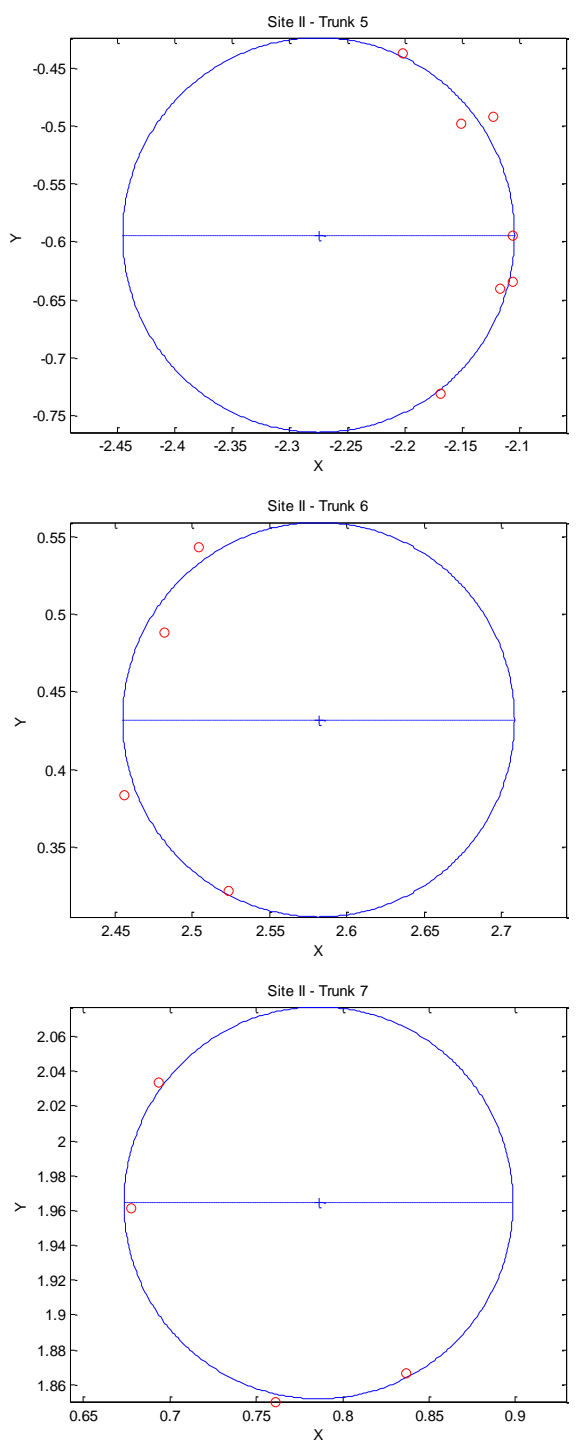

(b)

(c)

Figure 7. Circle fitted to the XY coordinates (small red circles) of points on the trunk surface.

To validate the results, each trunk was directly measured at the DBH (around $1.30 \mathrm{~m}$ of height) with a measuring tape. Table 2 presents the set of radii measured over seven trunks by manual measurement and the values indirectly estimated by the proposed technique. A comparison of results can be accomplished to assess the discrepancies based on the RMSE, which was approximately $1.8 \mathrm{~cm}$ with a standard deviation of around $0.7 \mathrm{~cm}$.

\begin{tabular}{c|c|c|c|c}
\hline Trunk & $\begin{array}{c}\text { Measured } \\
\text { radius } \\
(\mathrm{cm})\end{array}$ & $\begin{array}{c}\text { Indirectly } \\
\text { Estimated } \\
\text { radius } \\
(\mathrm{cm})\end{array}$ & $\begin{array}{c}\text { Estimated } \\
\text { standard } \\
\text { deviation } \\
\text { of the } \\
\text { radius } \\
(\mathrm{cm})\end{array}$ & $\begin{array}{c}\text { Discrepancy } \\
(\mathrm{cm})\end{array}$ \\
\hline 1 & 30.7 & 32.5 & 0.9 & -1.8 \\
2 & 15.1 & 12.8 & 0.8 & 2.3 \\
3 & 16.4 & 15.6 & 0.6 & 0.7 \\
4 & 21.8 & 21.2 & 0.2 & 0.6 \\
5 & 17.7 & 17.0 & 0.2 & 0.7 \\
6 & 13.7 & 11.3 & 0.1 & 2.4 \\
7 & 14.8 & 12.7 & 2.1 & 2.1 \\
\hline
\end{tabular}

Table 2. Comparison of results considering seven trunks: indirectly estimated radius against measured radius, around $1.30 \mathrm{~m}$ of height.

It is important to note the arrangement in Figure 6(a). The points have an unsuitable geometry that is approximately linear and may lead to an incorrect circle fitting. However, the solution was not affected due to the initial values assigned to the parameters $\mathrm{X}_{0}, \mathrm{Y}_{0}$ and $r$ in the adjustment process. For each trunk, the circle centre $\left(\mathrm{X}_{0}, \mathrm{Y}_{0}\right)$ was initially defined from the centre of mass of the estimated ground coordinates of the tie points, and $r$ was based on the largest standard deviation of these coordinates, adopting $r=2 \sigma$.

Ideally, points covering the whole width of the trunk are preferable to define the arc further. Another remark is concerning trunk 6 (in Figure 7(b) and Table 1). Its length has a vertical development with a certain inclination along the trunk. The resulting effect can be observed by the points projected to the ground, which resulted in a less accurate arc in comparison with the manual measurement.

In general, the results achieved with the proposed technique were compatible with the precision of the manual measurements, which may present errors above $1 \mathrm{~cm}$ due to variations on the trunk surface that affect the measurements. Therefore, the DBH value can be calculated for each tree using its respective estimated radius with the proposed technique.

\section{CONCLUSIONS}

A technique for the measurement of tree trunks was presented, and experiments were conducted to assess its feasibility using data acquired by a fisheye camera. The technique was based on bundle adjustment of multi-scale vertical images and considered trunks as regular cylindrical shapes over which some point coordinates were determined.

Trials were performed with a set of seven tree trunks from two sites in urban forest areas. The experiments demonstrated that multi-scale models can be oriented without control points, but using constraints imposed on the EOPs. A circle was geometrically adjusted to the planimetric coordinates of each trunk to determine its DBH. The analysis of results indicated discrepancies with a RMSE of $1.8 \mathrm{~cm}$ and standard deviation of $0.7 \mathrm{~cm}$, which is compatible with the measurements directly performed on the trunks. 
Based on the experiments, two concerns can be stated:

- Points extracted over the trunk surface should ideally cover the visible width of the trunk;

- Trunks with significant variations on their surfaces can complicate the generation of arcs on the plan when few points are used, but this problem can be solved with higher density of points extracted from the surface.

In future works, techniques will be developed to automate the dense point location over the tree trunks and to guarantee the best circle fitting for the calculation of the $\mathrm{DBH}$.

\section{REFERENCES}

Brown, D. C., 1971. Close-range calibration. Photogrammetric Engineering. 37(8), pp. 855-866.

Evans, G. D. and Coombe D. E.,1959. Hemispherical and woodland canopy photography and the light climate. Journal of Ecology. 47, pp. 103-113.

Forsman M; Börlin N. and Holmgren, J., 2012. Estimation of tree stem attributes using terrestrial photogrammetry. In: International archives of the photogrammetry, remote sensing and spatial information sciences, 2012. XXII ISPRS Congress. ISPRS International Society for Photogrammetry and Remote Sensing. Melbourne, Australia. v. XXXIX (Part B5), pp. 261265.

Garrido-Jurado, S.; Muñoz-Salinas, R.; Madrid-Cuevas, F.J. and Marín-Jiménez, M.J., 2014. Automatic generation and detection of highly reliable fiducial markers under occlusion. Pattern Recognition. 47(6), pp. 2280-2292.

Hapca, A. I.; Mothe, F. and Leban, J. M., 2007. A digital photographic method for 3D reconstruction of standing tree shape. Annals of forest science, 64(6), pp. 631-637.

Herrera P. J.; Pajares, G.; Guijarro, M.; Ruz J.J. and Cruz, J. M., 2011. A stereovision matching strategy for images captured with fish-eye lenses in forest environments. Sensors. 11(2), pp. 1756-1783.

Jonckheere, I.; Fleck, S.; Nackaerts, K.; Muys, B.; Coppin, P.; Weiss, M. and Baret, F., 2004. Review of methods for in situ leaf area index determination: Part I. Theories, sensors and hemispherical photography. Agricultural and Forest Meteorology. 121(1), pp. 19-35.

Lowe, D.G., 2004. Distinctive image features from scaleinvariant keypoints. International Journal of Computer Vision. 60(2), pp. 91-110.

Lowe, D.G. 2005. Demo Software: SIFT Keypoint Detector. http://www.cs.ubc.ca/ lowe/keypoints/ (25 Abr. 2014).

Ruy, R.; Tommaselli, A.M.G.; Galo, M.; Hasegawa, J.K. and Reis, T.T., 2009. Evaluation of bundle block adjustment with additional parameters using images acquired by SAAPI system. In: Proceedings of 6th International Symposium on Mobile Mapping Technology. Presidente Prudente, Brazil.

Schneider, D.; Schwalbe, E. and Maas, H.G., 2009. Validation of geometric models for fisheye lenses. ISPRS Journal of Photogrammetry and Remote Sensing. 64(3), pp. 259-266
Vaníček, P. and Wells, D. E., 1972. The Least Squares Approximation and Related Topics. Department of Surveying Engineering, University of New Brunswick. 69 p.

Tommaselli, A. M. G. and Berveglieri, A., 2014. Automatic orientation of multi-scale terrestrial images for 3D reconstruction. Remote Sensing. 6(4), pp. 3020-3040.

Tommaselli, A. M. G ; Marcato Junior, J.; Moraes, M.V.A.; Silva, S. L. A. and Artero, A. O., 2014. Calibration of panoramic cameras with coded targets and a 3D calibration field. In: Proceeding of EuroCOW 2014: the Calibration and Orientation Workshop, 2014, Castelldefels. v. 1. p. 1-6.

\section{ACKNOWLEDGMENTS}

The authors would like to thank the Fundação de Amparo à Pesquisa do Estado de São Paulo (FAPESP) for financial support - Grants 2010/16954-5, 2013/17787-3 and 2013/50426-4 . 